\title{
Time-Delay and Lavine's Formula
}

\section{Shu Nakamura}

Department of Pure and Applied Sciences, University of Tokyo, Komaba, Meguro-ku, Tokyo 153, Japan

\begin{abstract}
Lavine's results on time-delay ([10]) is extended to higher dimensional Schrödinger operators.
\end{abstract}

\section{Introduction}

In [10], Lavine proved the existence of a quantity called time-delay and gave its representation formula which we call "Lavine's formula," for one-dimensional Schrödinger operators. The aim of this paper is to extend them to $n$-dimensional Schrödinger operators.

We consider Schrödinger operators:

$$
H=H_{0}+V(x) ; \quad H_{0}=-\Delta
$$

on $\mathscr{H}=L^{2}\left(\mathbb{R}^{n}\right)$, and we suppose that the potential $V$ satisfies

Assumption $(V)$. $V(x)=V_{1}(x)+V_{2}(x)$, and there exists a constant $\varepsilon>0$ such that (i) $V_{1}(x)$ is a $C^{\infty}$-function and for any $\alpha$,

$$
\left|\left(\frac{\partial}{\partial x}\right)^{\alpha} V_{1}(x)\right| \leqq C_{\alpha}(1+|x|)^{-1-\varepsilon-|x|} ;
$$

(ii) the multiplication operator by $V_{2}(x)$ is compact from $H^{2}\left(\mathbb{R}^{n}\right)$ to $L^{2,2+\varepsilon}\left(\mathbb{R}^{n}\right)$.

$L^{2, \alpha}\left(\mathbb{R}^{n}\right)=\left\{\phi \in L_{\text {loc }}^{2}\left(\mathbb{R}^{n}\right):(1+|x|)^{\alpha} \phi \in L^{2}\left(\mathbb{R}^{n}\right)\right\}$ is the weighted $L^{2}$-space of order $\alpha$. Then, as is well-known, $H$ is self-adjoint; the wave operator defined by

$$
W_{ \pm}=\underset{t \rightarrow \pm \infty}{\mathrm{s}-\lim } \exp (i t H) \exp \left(-i t H_{0}\right)
$$

exists and is complete: $\operatorname{Ran} W_{ \pm}=\mathscr{H}^{a c}(H)$; hence the scattering operator defined by $S=W_{+}^{*} W_{-}$is unitary.

For $R>0$, let $X_{R}$ be a multiplication operator defined by

$$
\begin{gathered}
X_{R}=X_{R}(x) ; \quad X_{R}(x)=X(|x| / R) ; \quad 0 \leqq X(x) \leqq 1 ; \\
X \in C_{0}^{\infty}(\mathbb{R}) ; \quad X(x)=1 \quad \text { if }|x| \leqq 1,=0 \quad \text { if }|x| \geqq 2 .
\end{gathered}
$$


For $\phi, \psi \in \mathscr{H}$, we set $\phi(t)$ and $\phi_{0}(t)$ as

$$
\phi(t)=\exp (-i t H) W_{-} \phi ; \quad \phi_{0}(t)=\exp \left(-i t H_{0}\right) \phi
$$

and $\psi(t), \psi_{0}(t)$ similarly. Note that if $\phi \in H^{2}\left(\mathbb{R}^{n}\right), \phi(t)$ is the unique solution of the Schrödinger equation: $i(\partial / \partial t) \phi(t)=H \phi(t)$ such that $\left\|\phi(t)-\phi_{0}(t)\right\| \rightarrow 0(t \rightarrow-\infty)$.

We set $\mathscr{D}_{0}$ as

$$
\begin{aligned}
\mathscr{D}_{0}= & \left\{\phi \in \mathscr{H}: E_{H_{0}}(\Omega) \phi=\phi \quad \text { for some } \quad \Omega \subset(0, \infty) ;\right. \\
& \left.\Omega: \text { compact; } \Omega \cap \sigma_{p p}(H): \text { empty }\right\} .
\end{aligned}
$$

Then $T_{R}$ is defined by the following equation:

$$
\left(\phi, T_{R} \psi\right)=\int_{-\infty}^{\infty}\left(\phi(t), X_{R} \psi(t)\right) d t-\int_{-\infty}^{\infty}\left(\phi_{0}(t), X_{R} \psi_{0}(t)\right) d t
$$

for $\phi, \psi \in \mathscr{D}_{0}$. Since $X_{R}$ is $H_{0}$-smooth in the sense of Kato, and is local $H$-smooth in the sense of Lavine (see XIII-7, [11]), $\left(\phi, T_{R} \psi\right)$ exists for such $\phi$ and $\psi$, and bounded from below. Hence, $T_{R}$ is well-defined quadratic form on $\mathscr{D}_{0}$ and the Friedrichs extension exists. $T_{R}$ represents approximately the difference of the sojourn time of an interacting particle in the ball of radius $R$, and that of a free particle.

We set $\mathscr{D}_{1}=\mathscr{D}_{0} \cap L^{2,3}\left(\mathbb{R}^{n}\right)$, and $A=(1 / 2 i)(x \cdot(\partial / \partial x)+(\partial / \partial x) \cdot x)$ is the dilation generator. Our result is:

Theorem 1. Suppose Assumption $(V), \phi, \psi, S \phi$ and $S \psi \in \mathscr{D}_{1}$, then

$$
\lim _{R \rightarrow \infty}\left(\phi, T_{R} H_{0} \psi\right)=\int_{-\infty}^{\infty}\left(\phi(t),\left\{V+\frac{i}{2}[A, V]\right\} \psi(t)\right) d t .
$$

Of course, Theorem 1 implies that the limit of the L.H.S. and the integral of the R.H.S. exist, and are equal. It also asserts that the $\operatorname{limit:} \lim _{R \rightarrow \infty}\left(\phi, T_{R} \psi\right)$ exists for such $\phi$ and $\psi$. It is called time-delay.

On the other hand, in terms of the $S$-matrix $\{S(\lambda)\}$, the Eisenbud-Wigner timedelay operator is defined by

$$
T=\left\{-i S(\lambda) * \frac{d}{d \lambda} S(\lambda)\right\}
$$

on the spectral representation space for $H_{0}$. Jensen $([8,9])$ showed that under certain assumptions,

$$
\left(\phi, T H_{0} \psi\right)=\int_{-\infty}^{\infty}\left(\phi(t),\left\{V+\frac{i}{2}[A, V]\right\} \psi(t)\right) d t
$$

holds for $\phi, \psi \in \mathscr{D}_{0}$. Combining (1.7) with Theorem 1, we can conclude the following.

Theorem 2. If $\phi, \psi, S \phi$ and $S \phi \in \mathscr{D}_{1}$, then

$$
(\phi, T \psi)=\lim _{R \rightarrow \infty}\left(\phi, T_{R} \psi\right)
$$

This formula gives a relation between the $S$-matrix and the sojourn times of particles. 
Remark 1.1. By (1.6) and (1.7), the operator defined by the R.H.S. commutes with $H_{0}$. Hence if we set $\psi=H_{0}^{-1} \phi$ for $\phi \in \mathscr{D}_{1} \cap\left(S^{-1} \mathscr{D}_{1}\right)$, we have

$$
\lim _{R \rightarrow \infty}\left(\phi, T_{R} \phi\right)=\int_{-\infty}^{\infty}\left(H^{-1 / 2} \phi(t),\left\{V+\frac{i}{2}[A, V]\right\} H^{-1 / 2} \phi(t)\right) d t .
$$

As remarked by Lavine ([10]), if the quanity

$$
V+\frac{i}{2}[A, V]=V+\frac{i}{2} x \cdot \nabla V
$$

is non-positive everywhere, the time-delay is always non-positive, i.e. the interacting particles escape from every sufficiently large domain faster than the free particles.

Remark 1.2. We must consider how many $\phi$ 's such that $\phi \in \mathscr{D}_{1}$ and $S \phi \in \mathscr{D}_{1}$ exist because if such $\phi$ 's do not exist, Theorem 1 would be meaningless. But in many cases the set of such $\phi$ 's is dense in $\mathscr{H}$. For example, (i) if $V$ satisfies

$$
V: H^{2}\left(\mathbb{R}^{n}\right) \rightarrow L^{2,4+\varepsilon}\left(\mathbb{R}^{n}\right): \text { compact }
$$

for $\varepsilon>0$, then $\phi \in \mathscr{D}_{1}$ implies $S \phi \in \mathscr{D}_{1}$ (see Jensen [7]); (ii) if $V_{2}=0$ (i.e. $V$ is smooth and satisfies (1.1)), then $\phi \in \mathscr{D}_{1}$ and $\hat{\phi} \in C_{0}^{\infty}\left(\mathbb{R}^{n}\right)$ imply $S \phi \in \mathscr{D}_{1}$ and $(S \phi)^{\wedge} \in C_{0}^{\infty}\left(\mathbb{R}^{n}\right)$. This is a consequence of the result of Isozaki-Kitada [4]. It could also be proved that if $V_{2}$ satisfies (i) above, then $\phi \in \mathscr{D}_{1}$ and $\hat{\phi} \in C_{0}^{\infty}\left(\mathbb{R}^{n}\right)$ imply $S \phi \in \mathscr{D}_{1}$.

Time-delay has been studied by many physicists (see the introduction of Jensen [7] or Martin [13]) and mathematically rigorous treatment was initiated by Jauch and others $([5,6]$, see also [1]). In particular the time-dependent formulation of time-delay such as (1.4) was introduced by Jauch and Marchand [5]. Lavine ([10]) showed that (1.5) holds for one-dimensional Schrödinger operators with $V$ satisfying

$$
|V(x)|+\left|x \cdot V^{\prime}(x)\right| \leqq C(1+|x|)^{-1-\varepsilon} .
$$

Later, Jensen ([7]) proved that for $n$-dimensional Schrödinger operators (1.8) holds if $X_{R}$ is replaced by $X_{R}=E_{A}(\{\lambda:|\lambda|<R\})$. Jensen proved (1.7) also, which he called "Lavine's formula," under slightly weaker conditions than ours $([8,9])$. After this work was completed, the referee informed the author about papers of Wang $([14,16])$. He obtained similar results for smooth potential using a different method.

The outline of the proof is as follows: at first we construct a pseudo-differential operator $A_{R}$ such that

$$
X_{R} H_{0}=\frac{i}{2}\left[H_{0}, A_{R}\right]+(\text { small error terms })
$$

and that as $R \rightarrow \infty, A_{R} \rightarrow A+$ constant (Sect. 2); next, we introduce a operator $J_{ \pm}$ such that

$$
\begin{aligned}
\left\|\left(J_{ \pm}-1\right) e^{-i t H_{0}} \phi\right\| & \rightarrow 0 ; \\
\left\|\left(H J_{ \pm}-J_{ \pm} H_{0}\right) e^{-i t H_{0}} \phi\right\| & =O\left(t^{-2-\varepsilon}\right) ; \\
\left\|\left(J_{ \pm}^{*} J_{ \pm}-1\right) e^{-i t H_{0}} \phi\right\| & =O\left(t^{-1-\varepsilon}\right) \quad(t \rightarrow \pm \infty)
\end{aligned}
$$

(Sect. 3, cf. Isozaki-Kitada [4]); then minicking the proof of Lavine [10], we 
compute

$$
\left(\phi(t), X_{R} H \psi(t)\right)-\left(\phi_{0}(t), X_{R} H_{0} \psi_{0}(t)\right)
$$

and show that the error terms tend to zero as $R \rightarrow \infty$ (Sect. 4). For that purpose we employ the stationary phase method or the Enss method ([2]).

Notations. We shall use the following notations in the paper. We denote reals by $\mathbb{R}$ and Euclidean $n$-space by $\mathbb{R}^{n} . H^{s}\left(\mathbb{R}^{n}\right)$ is the Sobolev space of order $s$ and $L^{2, \alpha}\left(\mathbb{R}^{n}\right)$ is the weighted $L^{2}$-space of order $\alpha$. For Banach spaces $X$ and $Y, B(X, Y)$ denotes the Banach space of all bounded operators from $X$ to $Y$, and $B(X)=B(X, X)$.

We set $\langle x\rangle=\left(1+|x|^{2}\right)^{1 / 2} ; \hat{x}=x /|x|$ for $x \in \mathbb{R}^{n}$. We write any constant in the estimates by $C$ or $C_{*}$ denoting the dependence on $*$.

$\hat{\phi}$ denotes the Fourier transform of $\phi$, and for a symbol $a(x, \breve{\zeta}), x, \breve{\zeta} \in \mathbb{R}^{n}$, the operator $a\left(x, D_{x}\right)$ is defined by

$$
\left(a\left(x, D_{x}\right) \phi\right)(x)=(2 \pi)^{-n / 2} \int e^{i x \xi} a(x, \xi) \hat{\phi}(\xi) d \xi
$$

with $\phi \in \mathscr{S}$. About the theory of pseudo-differential operators, see e.g. Taylor [12] or Hörmander [3].

\section{Construction of the Operator $A_{R}$}

In this section, we construct the operator $A_{R}$ such that

$$
X_{R} H_{0} \sim \frac{i}{2}\left[H_{0}, A_{R}\right]
$$

in some sense. We set $A_{R} \sim a_{R}\left(x, D_{x}\right)$ with some symbol $a_{R}(x, \xi)$. Then, since

$$
\begin{aligned}
& \left(\frac{i}{2}\left[H_{0}, a_{R}\left(x, D_{x}\right)\right] \phi\right)(x) \\
& \quad=(2 \pi)^{-n / 2} \int e^{i x \xi}\left\{\xi \partial_{x} a_{R}(x, \xi)-\frac{i}{2} \Delta_{x} a_{R}(x, \xi)\right\} \hat{\phi}(\xi) d \xi,
\end{aligned}
$$

(2.1) formally implies

$$
\xi \partial_{x} a_{R}(x, \xi)-\frac{i}{2} \Delta_{x} a_{R}(x, \xi) \sim X_{R}(x) \xi^{2} .
$$

We solve (2.2) as follows: let $a_{R}(x, \xi)=a_{R}^{(0)}(x, \xi)+a_{R}^{(1)}(x, \xi)$ and these are solutions of the equations

$$
\begin{gathered}
\xi \partial_{x} a_{R}^{(0)}(x, \xi)=X_{R}(x) \xi^{2} \\
\xi \partial_{x} a_{R}^{(1)}(x, \xi)=\frac{i}{2} \Delta_{x} a_{R}^{(0)}(x, \xi) .
\end{gathered}
$$

Then the remainder term is

$$
\xi \partial_{x} a_{R}-\frac{i}{2} \Delta_{x} a_{R}-X_{R} \xi^{2}=-\frac{i}{2} \Delta_{x} a_{R}^{(1)}(x, \xi) \equiv b_{R}(x, \xi) .
$$


Transport equations (2.3), (2.4) can be easily solved if $\xi \neq 0$, and we choose the following solutions:

$$
\begin{aligned}
a_{R}^{(0)}(x, \xi) & =-\int_{0}^{\infty} \xi^{2} X_{R}(x+t \xi) d t+\int_{0}^{\infty} \xi^{2} X_{R}(t \xi) d t \\
& =|\xi|\left\{-\int_{0}^{\infty} X_{R}(x+t \hat{\xi}) d t+\int_{0}^{\infty} X_{R}(t \hat{\xi}) d t\right\} \\
& =-|\xi| \int_{0}^{\infty} d t \int_{0}^{1} d \theta x \cdot\left(\nabla X_{R}\right)(\theta x+t \hat{\xi}) ; \\
a_{R}^{(1)}(x, \xi) & =\frac{i}{2} \int_{0}^{\infty}\left\{|\xi| \int_{0}^{\infty}\left(\Delta_{x} X_{R}\right)(x+t \hat{\xi}+s \hat{\xi}) d t\right\} \frac{d s}{|\xi|} \\
& =\frac{i}{2} \int_{0}^{\infty}\left(\Delta X_{R}\right)(x+s \hat{\xi}) s d s ; \\
b_{R}(x, \xi) & =\frac{1}{4} \int_{0}^{\infty}\left(\Delta^{2} X_{R}\right)(x+s \hat{\xi}) s d s .
\end{aligned}
$$

Lemma 2.1. $a_{R}^{(0)}(x, \xi)$ and $a_{R}^{(1)}(x, \xi)$ are the unique solutions of $(2.3)$ and (2.4) such that for $\xi \neq 0, a_{R}^{(0)}(0, \xi)=0$ and

$$
\begin{aligned}
& a_{R}^{(0)}(x, \xi)=|\xi| \int_{0}^{\infty} X_{R}(t \hat{\xi}) d t=|\xi| \times \text { constant } \times R \\
& a_{R}^{(1)}(x, \xi)=0
\end{aligned}
$$

if $|x| \geqq 2 R$ and $x \cdot \xi \geqq 0$.

This can be verified directly. We next consider their asymptotic properties as $R \rightarrow \infty$.

Proposition 2.1. For $(x, \xi) \in \mathbb{R}^{n} \times\left(\mathbb{R}^{n} /\{0\}\right), a_{R}^{(0)}(x, \xi) \rightarrow x \cdot \xi ; a_{R}^{(1)}(x, \xi) \rightarrow-(i / 2)(n-2)$; $b_{R}(x, \xi) \rightarrow 0$, as $R \rightarrow \infty$, locally uniformly.

Proof. We may suppose $\hat{\xi}=(1,0,0, \ldots, 0)$, and we write

$$
x=\left(x_{1}, x^{\prime}\right) \in \mathbb{R} \times \mathbb{R}^{n-1} ; \quad \nabla^{\prime}=\frac{\partial}{\partial x^{\prime}} ; \quad \Delta^{\prime}=\nabla^{\prime} \cdot \nabla^{\prime} .
$$

By (2.5), we have

$$
\begin{aligned}
a_{R}^{(0)}(x, \xi)= & -|\xi|\{(x, \hat{\xi}) \hat{\xi}+(x-(x, \hat{\xi}) \hat{\xi})\} \int_{0}^{\infty} d s \int_{0}^{1} d \theta \nabla X_{R}(\theta x+s \hat{\xi}) \\
= & -x \cdot \xi \int_{0}^{\infty} d s \int_{0}^{1} d \theta \frac{d}{d s}\left\{X_{R}(\theta x+s \hat{\xi})\right\} \\
& -|\xi| x^{\prime} \cdot \int_{0}^{\infty} d s \int_{0}^{1} d \theta\left(\nabla^{\prime} X_{R}\right)(\theta x+s \hat{\xi}) .
\end{aligned}
$$


Since

$$
\int_{0}^{\infty} d s \int_{0}^{1} d \theta \frac{d}{d s}\left\{X_{R}(\theta x+s \hat{\xi})\right\}=-\int_{0}^{1} d \theta X_{R}(\theta x)=-1
$$

If $R \geqq|x|$, combining this with (2.8), we obtain

$$
a_{R}^{(0)}(x, \xi)=x \cdot \xi+|\xi| \int_{0}^{1} d \theta \int_{0}^{\infty} d s x^{\prime} \cdot \nabla^{\prime} X_{R}(\theta x+s \hat{\xi}) .
$$

We shall show that the second term converges to zero as $R \rightarrow \infty$. Set $y=\theta x+s \hat{\xi}$, $r=|y|$. By definition of $X_{R},(1.2)$, we see

$$
\left(\nabla X_{R}\right)(y)=\frac{\partial r}{\partial y} \frac{\partial}{\partial r} X_{R}=\frac{y}{r} \frac{1}{R} X^{\prime}(r / R) .
$$

On the other hand, on the support of $\nabla X_{R}(y),(y / r)=\hat{\xi}+O\left(R^{-1}\right)$ for each $x$ and $\theta$. Hence

$$
\begin{gathered}
x^{\prime} \cdot \nabla^{\prime} X_{R}(y)=O\left(R^{-1}\right) \cdot \frac{1}{R} \cdot X^{\prime}(y / R)=O\left(R^{-2}\right), \\
\left|\int_{0}^{1} d \theta \int_{0}^{\infty} d s x^{\prime} \cdot \nabla^{\prime} X_{R}(y)\right| \leqq R \cdot C \cdot R^{-2} \leqq C \cdot R^{-1} .
\end{gathered}
$$

This completes the proof of $a_{R}^{(0)}(x, \xi) \rightarrow x \cdot \xi$.

By (2.6), we have

$$
a_{R}^{(1)}(x, \xi)=\frac{i}{2} \int_{0}^{\infty}\left(\frac{\partial}{\partial x_{1}}\right)^{2} X_{R}(x+t \hat{\xi}) t d t+\frac{i}{2} \int_{0}^{\infty}\left(\Delta^{\prime} X_{R}(x+t \hat{\xi}) t d t .\right.
$$

By integration by parts, the first term is

$$
\begin{aligned}
& \frac{i}{2} \int_{0}^{\infty}(\hat{\xi} \cdot \nabla)^{2} X_{R}(x+t \hat{\xi}) t d t=\frac{i}{2} \int_{0}^{\infty} \frac{d}{d t}\left\{\hat{\xi} \cdot \nabla X_{R}(x+t \hat{\xi})\right\} t d t \\
& \quad=-\frac{i}{2} \int_{0}^{\infty} \hat{\xi} \nabla X_{R}(x+t \hat{\xi}) d t=-\frac{i}{2} \int_{0}^{\infty} \frac{d}{d t}\left\{X_{R}(x+t \hat{\xi})\right\} d t \\
& \quad=\frac{i}{2} X_{R}(x) .
\end{aligned}
$$

By elementary calculations, one can obtain

$$
\begin{aligned}
\Delta^{\prime} X_{R}(y) & =\Delta^{\prime} r \frac{d}{d r} X_{R}+\left|\nabla^{\prime} r\right|^{2} \frac{d^{2}}{d r^{2}} X_{R} \\
& =\left(\frac{n-1}{r}-\frac{\left|y^{\prime}\right|^{2}}{r^{3}}\right) R^{-1} X^{\prime}\left(\frac{|y|}{R}\right)+\frac{\left|y^{\prime}\right|^{2}}{r^{2}} R^{-2} X^{\prime \prime}\left(\frac{|y|}{R}\right),
\end{aligned}
$$

where $y=x+t \hat{\xi}$ and $r=|y|$. Since on the support of $\nabla X_{R}$

$$
\frac{d r}{d t}=\frac{(x+t \hat{\xi}) \hat{\xi}}{r}=\frac{t}{r}+\frac{x \cdot \hat{\xi}}{r}=\frac{t}{r}+O\left(R^{-1}\right),
$$


and $\left|y^{\prime}\right|=\left|x^{\prime}\right|$, we have

$$
\begin{aligned}
\Delta^{\prime} X_{R}(y) \cdot t & =(n-1)(t / r) R^{-1} X^{\prime}(r / R)+O\left(R^{-3}\right) \\
& =(n-1) \frac{d r}{d t} R^{-1} X^{\prime}(r / R)+O\left(R^{-2}\right) \\
& =(n-1) \frac{d r}{d t} \frac{d}{d r} X_{R}(r)+O\left(R^{-2}\right) \\
& =(n-1) \frac{d}{d t} X_{R}(y)+O\left(R^{-2}\right) .
\end{aligned}
$$

Hence, the second term of (2.9) is

$$
\frac{i}{2}(n-1) \int_{0}^{\infty} \frac{d}{d t} X_{R}(y) d t+O\left(R^{-1}\right)=-\frac{i}{2}(n-1) X_{R}(x)+O\left(R^{-1}\right) .
$$

Combining this with (2.10), we conclude

$$
a_{R}^{(1)}(x, \xi)=-\frac{i}{2}(n-2) X_{R}(x)+O\left(R^{-1}\right) .
$$

$b_{R}(x, \xi) \rightarrow 0$ can be shown easily from $(2.7)$ since $\left(\Delta^{2} X_{R}\right)(y)=O\left(R^{-4}\right)$.

Let $\rho \in C^{\infty}([-1,1])$ such that $0 \leqq \rho(x) \leqq 1 ; \rho(x)=1$ if $x \leqq 1 / 4,=0$ if $x \leqq-1 / 4$. We set

$$
\begin{aligned}
Y_{R}(x, \xi) & =X_{2 R}(x)+\left(1-X_{2 R}(x)\right) \rho(\hat{x} \cdot \hat{\xi}) \\
& =X\left(\frac{|x|}{2 R}\right)+\left(1-X\left(\frac{|x|}{2 R}\right)\right) \rho\left(\frac{x}{|x|} \cdot \frac{\xi}{|\xi|}\right),
\end{aligned}
$$

and define $\tilde{a}_{R}(x, \xi)$ and $c_{R}(x, \xi)$ by

$$
\begin{gathered}
\tilde{a}_{R}(x, \xi)=a_{R}(x, \xi) Y_{R}(x, \xi) \\
c_{R}(x, \xi)=\xi \partial_{x} \tilde{a}_{R}(x, \xi)-\frac{i}{2} \Delta_{x} \tilde{a}_{R}(x, \xi)-X_{R}(x) \xi^{2} .
\end{gathered}
$$

By easy computations, we obtain

$$
c_{R}=b_{R} Y_{R}+a_{R} \xi \cdot \partial_{x} Y_{R}-i \partial_{x} a_{R} \cdot \partial_{x} Y_{R}-\frac{i}{2} a_{R}\left(\Delta_{x} Y_{R}\right)
$$

Lemma 2.2. For each $\alpha, \beta, \delta>0$,

(i) $\left|\partial_{x}^{\alpha} \partial_{\xi}^{\beta} \tilde{a}_{R}(x, \xi)\right| \leqq C_{\alpha \beta \delta} \min \left(\langle x\rangle^{-|x|}, R\right)\langle\xi\rangle^{1-|\beta|}(|\xi|>\delta)$;

(ii) $\left|\partial_{x}^{\alpha} \partial_{\xi}^{\beta} c_{R}(x, \xi)\right| \leqq\left(\begin{array}{ll}C_{\alpha \beta \delta}\langle x\rangle^{-|\alpha|}\langle\xi\rangle^{1-|\beta|} & (|\xi|>\delta) \\ C_{\alpha \beta \delta}\langle x\rangle^{-2-|\alpha|}\langle\xi\rangle^{-|\beta|} & \left(|\xi|>\delta, \hat{x} \cdot \hat{\xi}>-\frac{1}{4}\right),\end{array}\right.$

where $C_{\alpha \beta \delta}$ 's are independent of $R$.

Proof. By (2.5) and (2.6), if $|x| \leqq 4 R$ or $\hat{x} \cdot \hat{\xi} \geqq-1 / 4$,

$$
\left|\partial_{x}^{\alpha} \partial_{\xi}^{\beta} a_{R}^{(0)}(x, \xi)\right| \leqq\left(\begin{array}{ll}
C_{\beta} \min (|x|, R)|\xi|^{1-|\beta|} & (\alpha=0) \\
C_{\alpha \beta} R^{1-|\alpha|}|\xi|^{1-|\beta|} & (\alpha \neq 0),
\end{array}\right.
$$




$$
\left|\partial_{x}^{\alpha} \partial_{\xi}^{\beta} a_{R}^{(1)}(x, \xi)\right| \leqq C_{\alpha \beta} R^{-|\alpha|}|\xi|^{-|\beta|} .
$$

Since $\operatorname{supp}\left(\partial_{x}^{\alpha} a_{R}^{(i)}\right) \subset\{(x, \xi):|x| \leqq 2 \sqrt{2}$ or $\hat{x} \cdot \hat{\xi} \leqq-1 / \sqrt{2}\}$ if $\alpha \neq 0$ or $i=1$, we have

$$
\left|\partial_{x}^{\alpha} \partial_{\xi}^{\beta} a_{R}^{(i)}(x, \xi)\right| \leqq C_{\alpha \beta} \min \left(\langle x\rangle^{1-i-|\alpha|}, R\right)|\xi|^{1-i-|\beta|}
$$

on $\operatorname{supp} Y_{R}$, for $i=0,1$. By the definition of $Y_{R}$, we have also

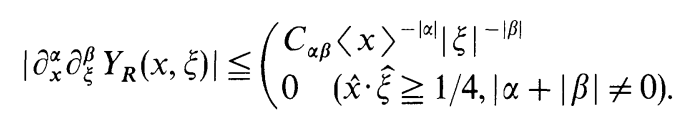

Then (i) follows easily from these estimates.

By (2.7), we obtain

$$
\begin{aligned}
\left|\partial_{x}^{\alpha} \partial_{\xi}^{\beta} b_{R}(x, \xi)\right| & \leqq C_{\alpha \beta} R^{-2-|\alpha|}|\xi|^{-|\beta|} \\
& \leqq C_{\alpha \beta}\langle x\rangle^{-2-|\alpha|}|\xi|^{-|\beta|},
\end{aligned}
$$

similarly if $|x| \leqq 4 R$ or $\hat{x} \cdot \hat{\xi} \geqq-1 / 4$. Hence

$$
\begin{aligned}
& \left|\partial_{x}^{\alpha} \partial_{\xi}^{\beta}\left(b_{R} Y_{R}\right)(x, \xi)\right| \leqq C\langle x\rangle^{-2-|\alpha|}|\xi|^{-|\beta|} ; \\
& \left|\partial_{x}^{\alpha} \partial_{\xi}^{\beta}\left(a_{R} \xi \cdot \partial_{x} Y_{R}\right)(x, \xi)\right| \leqq\left(\begin{array}{ll}
C\langle x\rangle^{-|\alpha|}|\xi|^{2-|\beta|} \\
0 & (\hat{x} \cdot \hat{\xi} \geqq 1 / 4) ;
\end{array}\right. \\
& \left|\partial_{x}^{\alpha} \partial_{\xi}^{\beta}\left(\partial_{x} a_{R} \cdot \partial_{x} Y_{R}\right)(x, \xi)\right| \leqq\left(\begin{array}{l}
C\langle x\rangle^{-1-|\alpha|}|\xi|^{1-|\beta|} \\
0 \quad(\hat{x} \cdot \hat{\xi} \geqq 1 / 4) ;
\end{array}\right. \\
& \left|\partial_{x}^{\alpha} \partial_{\xi}^{\beta}\left(a_{R} \Delta_{x} Y_{R}\right)(x, \xi)\right| \leqq\left(\begin{array}{l}
C\langle x\rangle^{-1-|\alpha|}|\xi|^{1-|\beta|} \\
0 \quad(\hat{x} \cdot \hat{\xi} \geqq 1 / 4)
\end{array}\right.
\end{aligned}
$$

using (2.13) again. Equation (2.11) and these estimates imply (ii).

Since our symbols have singularities at $\xi=0$, we must introduce a suitable cutoff. We set $Z_{R}$ as

$$
\begin{aligned}
& Z_{R}=Z_{R}\left(D_{x}\right) ; \quad Z_{R}(\xi)=Z(R|\xi|) ; \\
& Z \in C^{\infty}(\mathbb{R}) ; \quad 0 \leqq Z(\xi) \leqq 1(\xi \in \mathbb{R}) ; \\
& Z(\xi)=0 \quad \text { if } \quad|\xi| \leqq 1,=1 \quad \text { if } \quad|\xi| \geqq 2 .
\end{aligned}
$$

We define $A_{R}$ and $C_{R}$ by

$$
\begin{array}{ll}
A_{R}(x, \xi)=\tilde{a}_{R}(x, \xi) Z_{R}(\xi) ; & A_{R}=A_{R}\left(x, D_{x}\right), \\
C_{R}(x, \xi)=c_{R}(x, \xi) Z_{R}(\xi) ; & C_{R}=C_{R}\left(x, D_{x}\right) .
\end{array}
$$

Then, by Lemma 2.3 and the $L^{2}$-boundedness theorem (Ch. 13 of [12]), $A_{R}$ is in $B\left(H^{1}\left(\mathbb{R}^{n}\right), L^{2}\left(\mathbb{R}^{n}\right)\right)$, and $C_{R}$ is in $B\left(H^{2}\left(\mathbb{R}^{n}\right), L^{2}\left(\mathbb{R}^{n}\right)\right)$ for each $R$. Moreover, we can prove their uniform boundedness in $R$.

\section{Proposition 2.2.}

(i) $\sup _{R \geqq 1}\left\|A_{R}\right\|_{B\left(H^{1}\left(\mathbb{R}^{n}\right), L^{2,-1}\left(\mathbb{R}^{n}\right)\right)}<\infty$,

(ii) $\sup _{R \geq 1}\left\|A_{R}^{*}\right\|_{B\left(H^{1}\left(\mathbb{R}^{n}\right), L^{2,-1}\left(\mathbb{R}^{n}\right)\right)}<\infty$, 
(iii) $\sup _{R \geq 1}\left\|C_{R}\right\|_{B\left(H^{2}\left(\mathbb{R}^{n}\right), L^{2}\left(\mathbb{R}^{n}\right)\right)}<\infty$,

(iv) $\sup _{R \geqq 1}\left\|C_{R}^{*}\right\|_{B\left(H^{2}\left(\mathbb{R}^{n}\right), L^{2}\left(\mathbb{R}^{n}\right)\right)}<\infty$.

Proof. We define $d_{R, k}(x, \xi)$ by

$$
d_{R, k}(x, \xi)=-\int_{0}^{\infty} d s \int_{0}^{1} d \theta \frac{\partial}{\partial x_{k}} X_{R}(\theta x+s \hat{\xi}) Y(x, \xi)
$$

for $k=1, \ldots, n$, then by $(2.5)$ we see

$$
a_{R}^{(0)}(x, \xi) Y_{R}(x, \xi)=\sum_{k} x^{k} d_{R, k}(x, \xi)|\xi| .
$$

By a change of coordinates, one immediately obtains $d_{R, k}(x, \xi) Z_{R}(\xi)=$ $d_{1, k}(x / R, R \xi) Z_{1}(R \xi)$. Hence, if we set $\rho=\log R$ and $U(\sigma)$ be the dilation operator defined by $(U(\sigma) \phi)(x)=\exp (n \sigma / 2) \phi\left(e^{\sigma} x\right)(\sigma \in \mathbb{R})$, we have

$$
\begin{aligned}
d_{R, k}\left(x, D_{x}\right) Z_{R}\left(D_{x}\right) & =d_{1, k}\left(x / R, R \cdot D_{x}\right) Z_{1}\left(R \cdot D_{x}\right) \\
& =U(-\rho) d_{1, k}\left(x, D_{x}\right) Z_{1}\left(D_{x}\right) U(\rho) .
\end{aligned}
$$

Since $U(\rho)$ is unitary, $\left\|d_{R, k}\left(x, D_{x}\right) Z_{R}\right\|_{B(\mathscr{H})}=$ constant (we remarked that $d_{1, k}\left(x, D_{x}\right) Z_{1}\left(D_{x}\right)$ is bounded in $\left.L^{2}\left(\mathbb{R}^{n}\right)\right)$. This and (2.14) yield

$$
\left\|\left(a_{R}^{(0)} Y_{R} Z_{R}\right)\left(x, D_{x}\right)\right\|_{B\left(H^{1}, L^{2,-1}\right)} \leqq C \text {. }
$$

On the other hand, $a_{R}^{(1)}(x, \xi)=a_{1}^{(1)}(x / R, R \xi)$ and an analogous argument can be carried out to show the uniform boundedness of $\left(a_{R}^{(1)} Y_{R} Z_{R}\right)\left(x, D_{x}\right)$ in $B(\mathscr{H})$. These imply (i).

Next, by the definition and (2.14), we see

$$
\begin{aligned}
\left(\left(a_{R}^{(0)} Y_{R} Z_{R}\right)(x,\right. & \left.\left.D_{x}\right)\right)^{*} \phi(x) \\
= & (2 \pi)^{-n} \sum_{k} \int^{i(x-y) \xi} d_{R, k}(y, \xi)|\xi| Z_{R}(\xi) y_{k} \phi(y) d y d \xi \\
= & (2 \pi)^{-n} \sum_{k} \int_{\partial} \frac{\partial}{\partial \xi_{k}}\left\{e^{i(x-y) \xi}\right\} d_{R, k}(y, \xi)|\xi| Z_{R}(\xi) \phi(y) d y d \xi \\
& -(2 \pi)^{-n} \sum_{k} i x_{k} \int e^{i(x-y) \xi} d_{R, k}(y, \xi)|\xi| Z_{R}(\xi) \phi(y) d y d \xi \\
= & \mid \phi+\llbracket \phi,
\end{aligned}
$$

and by integration by parts,

$$
\begin{aligned}
\mid \phi= & -i(2 \pi)^{-n} \sum_{k} \int e^{i(x-y) \xi} \frac{\partial}{\partial \xi_{k}}\left\{d_{R, k}(y, \xi)|\xi| Z_{R}(\xi)\right\} \phi(y) d y d \xi \\
= & -i(2 \pi)^{-n} \sum_{k} \int e^{i(x-y) \xi} \frac{\partial}{\partial \xi_{k}}\left\{d_{R, k}(y, \xi)|\xi|\right\} Z_{R}(\xi) \phi(y) d y d \xi \\
& -(2 \pi)^{-n} \sum_{k} \int e^{i(x-y) \xi} d_{R, k}(y, \xi)|\xi| \frac{\partial}{\partial \xi_{k}}\left\{Z_{R}(\xi)\right\} \phi(y) d y d \xi \\
= & I_{1} \phi+I_{2} \phi .
\end{aligned}
$$


For $\mathrm{I}_{1}$, one can prove the uniform boundedness in $B(\mathscr{H})$ by the same method as above. Since

$$
\mathrm{I}_{2} \phi=-i \sum_{k}\left\{\left|D_{x}\right|\left(\frac{\partial}{\partial \xi_{k}} Z_{R}\right)\left(D_{x}\right)\right\} d_{R, k}\left(x, D_{x}\right)^{*} \phi,
$$

and the symbol of $\left\{\left|D_{x}\right|\left(\partial / \partial \xi_{k} Z_{R}\right)\left(D_{x}\right)\right\}$ is bounded uniformly in $\xi$ and $R, \mathrm{I}_{2}$ is uniformly bounded in $B(\mathscr{H})$.

$$
\begin{aligned}
\square \phi= & -i(2 \pi)^{-n} \sum_{k, j} x_{k} \int e^{i(x-y) \xi} d_{R, k}(y, \xi) Z_{R}(\xi) \xi_{j} \hat{\xi}_{j} \phi(y) d y d \xi \\
= & (2 \pi)^{-n} \sum_{k, j} x_{k} \int \frac{\partial}{\partial y_{j}}\left\{e^{i(x-y) \xi}\right\} d_{R, k}(y, \xi) Z_{R}(\xi) \hat{\xi}_{j} \phi(y) d y d \xi \\
= & -(2 \pi)^{-n} \sum_{k, j} x_{k} \int e^{i(x-y) \xi} \frac{\partial}{\partial y_{j}} d_{R, k}(y, \xi) Z_{R}(\xi) \hat{\xi}_{j} \phi(y) d y d \xi \\
& -(2 \pi)^{-n} \sum_{k, j} x_{j} \int e^{i(x-y) \xi} d_{R, k}(y, \xi) Z_{R}(\xi) \hat{\xi}\left\{\frac{\partial}{\partial y_{j}} \phi(y)\right\} d y d \xi \\
= & \left.-\sum_{k, j} x_{k}\left\{\left(\frac{\partial}{\partial x_{j}} d_{R, k}\right)\left(x, D_{x}\right) Z_{R}\left(\hat{D}_{x}\right)_{j}\right\}\right\}^{*} \phi \\
& -\sum_{k, j} x_{k}\left\{d_{R, k}\left(x, D_{x}\right) Z_{R}\left(\hat{D}_{x}\right)_{j}\right\}^{*} \frac{\partial}{\partial x_{j}} \phi \\
= & \mathbb{1}_{1} \phi+\mathbb{0}_{2} \phi .
\end{aligned}
$$

Similar argument shows that $\left\{\left(\left(\partial / \partial x_{j}\right) d_{R, k}\right)\left(x, D_{x}\right) Z_{R} \hat{D}_{x}\right\}^{*}$ and $\left\{a_{R . k}^{(0)}\left(x, D_{x}\right) Z_{R} \hat{D}_{x}\right\}^{*}$ are uniformly bounded in $B(\mathscr{H})$, again. Hence $\nabla_{1}$ is uniformly bounded in $B\left(\mathscr{H}, L^{2 \cdot-1}\right)$, and $\rrbracket_{2}$ is uniformly bounded in $B\left(H^{1}, L^{2,1}\right)$. These prove (ii).

The next estimates can be shown in the same way:

$$
\begin{aligned}
\left\|\left(b_{R} Y_{R} Z_{R}\right)\left(x, D_{x}\right)\right\|_{B(\mathscr{H})} & \leqq C R^{-2} ; \\
\left\|\left(a_{R} \xi \cdot \partial_{x} Y_{R}\right)\left(x, D_{x}\right)\right\|_{B\left(H^{2}, L^{2}\right)} & \leqq C ; \\
\left\|\left(\partial_{x} a_{R} \cdot \partial_{x} Y_{R}\right)\left(x \cdot D_{x}\right)\right\| E_{B\left(H^{1}, L^{2}\right)} & \leqq C R^{-1} ; \\
\left\|\left(a_{R} \Delta_{x} Y_{R}\right)\left(x, D_{x}\right)\right\|_{B\left(H^{1}, L^{2}\right)} & \leqq C R^{-1},
\end{aligned}
$$

and (iii) follows. (iv) can be proved by the standard method using integration by parts.

Remark 2.1. Using the Calderon-Lions interpolation theorem (Th. IX-20 of [11]), one can prove that $A_{R}\left(C_{R}\right.$ respectively) is uniformly bounded in $B\left(H^{s, \alpha}\left(\mathbb{R}^{n}\right), H^{s-1, \alpha-1}\left(\mathbb{R}^{n}\right)\right) \quad\left(B\left(H^{s+2}, H^{s}\right)\right.$ respectively) for $s \in \mathbb{R}, 0 \leqq \alpha \leqq 1$, where $H^{s, \alpha}\left(\mathbb{R}^{n}\right)$ is the weighted Sobolev space.

The next lemma follows easily from the definitions, (2.11) and Proposition 2.1:

Lemma 2.3. For $(x, \xi) \in \mathbb{R}^{n} \times\left(\mathbb{R}^{n} /\{0\}\right), A_{R}(x, \xi) \rightarrow x \cdot \xi-(i / 2)(n-2) ; C_{R} \rightarrow 0$ as $R \rightarrow \infty$, locally uniformly. 


\section{Modifier $\boldsymbol{J}_{ \pm}$}

Here we introduce a pseudo-differential operator $J_{ \pm}$such that it satisfies (1.9). $J_{ \pm}$we shall define is approximately the same as that in Isozaki-Kitada [4] (for short range potentials), and their modifier is more precise than ours. But our construction is slightly easier to handle and enough for our purpose.

Let $p_{ \pm}(x, \xi)$ be a solution of

$$
\begin{aligned}
2 i \xi \cdot \partial_{x} p_{ \pm}(x, \xi) & =V_{1}(x): \\
\sigma_{ \pm}(x, \xi)=-\frac{1}{2 i} \int_{0}^{ \pm \infty} V_{1}(x+t \xi) d t & =-\frac{1}{2 i} \frac{1}{|\xi|} \int_{0}^{ \pm \infty} V_{1}(x+t \hat{\xi}) d t .
\end{aligned}
$$

It satisfies

$$
\left|\partial_{x}^{\alpha} \partial_{\xi}^{\beta} p_{ \pm}(x, \xi)\right| \leqq C_{\alpha \beta}|\xi|^{-1-|\beta|}\langle x\rangle^{-\varepsilon-|\alpha|},
$$

If $\pm \hat{x} \cdot \hat{\xi} \geqq-1 / 4$. We set $j_{ \pm}(x, \xi)=j_{ \pm}(\delta, \Delta ; x, \xi)(0<\delta<\Delta<\infty)$ by

$$
j_{ \pm}(x, \xi)=\exp \left\{p_{ \pm}(x, \xi)\left(1-X_{1}(x)\right) \rho( \pm \hat{x} \cdot \hat{\xi})\right\} f\left(\delta, \Delta ;|\xi|^{2}\right),
$$

where $f(\delta, \Delta ; \lambda) \in C_{0}^{\infty}((O, \infty)) ; 0 \leqq f(\delta, \Delta ; \lambda) \leqq 1 ; f(\delta, \Delta ; \lambda)=1 \quad$ if $\quad \lambda \in[\delta, \Delta],=0$ if $\lambda \notin(\delta / 2,2 \Delta)$. Let $t_{ \pm}(x, \xi)=t_{ \pm}(\delta, \Delta ; x, \xi)$ be

$$
t_{ \pm}(x, \xi)=2 i \xi \cdot \partial_{x} j_{ \pm}(x, \xi)+\Delta_{x} j_{ \pm}(x, \xi)+V_{1}(x) j_{ \pm}(x, \xi) .
$$

Lemma 3.1. For any $\alpha, \beta$,

(i) $\left|j_{ \pm}(x, \xi)\right| \leqq 1$;

(ii) $\left|\partial_{x}^{\alpha} \partial_{\beta}^{\alpha} j_{ \pm}(x, \xi)\right| \leqq C_{\alpha \beta}\langle x\rangle^{-t-|\alpha|} \quad(|\alpha|+|\beta| \neq 0)$;

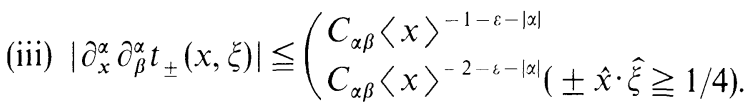

Proof. (i) is immediate since $p_{ \pm}(x, \xi)$ is pure imaginary. (ii) follows from (3.3) and the fact that $\rho( \pm \hat{x} \cdot \hat{\xi})$ is homogeneous in $x$. By (3.3),

$$
\begin{aligned}
t_{ \pm}(x, \xi)= & 2 i \xi \cdot \partial_{x}\left\{p_{ \pm}(x, \xi)\left(1-X_{1}(x)\right) \rho( \pm \hat{x} \cdot \hat{\xi})\right\} j_{ \pm}(x, \xi) \\
& +\Delta_{x}\left\{p_{ \pm}(x, \xi)\left(1-X_{1}(x)\right) \rho( \pm \hat{x} \cdot \hat{\xi})\right\} j_{ \pm}(x, \xi) \\
& +\left|\partial_{x}\left\{p_{ \pm}(x, \xi)\left(1-X_{1}(x)\right) \rho( \pm \hat{x} \cdot \hat{\xi})\right\}\right|^{ \pm} j_{ \pm}(x, \xi) \\
& -V_{1}(x) j_{ \pm}(x, \xi) \\
= & \left\{2 i \xi \cdot \partial_{x} p_{ \pm}(x, \xi)-V_{1}(x)\right\} j_{ \pm}(x, \xi) \\
& +2 i \xi \cdot \partial_{x}\left\{\left(1-X_{1}(x)\right) \rho( \pm \hat{x} \cdot \hat{\xi})\right\} p_{ \pm}(x, \xi) j_{ \pm}(x, \xi) \\
& +\Delta_{x}\left\{p_{ \pm}(x, \xi)\left(1-X_{1}(x)\right) \rho( \pm \hat{x} \cdot \hat{\xi})\right\} j_{ \pm}(x, \xi) \\
& +\left|\partial_{x}\left\{p_{ \pm}(x, \xi)\left(1-X_{1}(x)\right) \rho( \pm \hat{x} \cdot \hat{\xi})\right\}\right|^{2} j_{ \pm}(x, \xi) .
\end{aligned}
$$

The first term vanishes by (3.1), and it is easily seen that the second term satisfies the former property of (iii), and vanishes outside $\{x:|x| \leqq 2\}$ if $\pm \hat{x} \cdot \hat{\xi} \geqq 1 / 4$. (iii) follows since the third term is $O\left(\langle x\rangle^{-2-\varepsilon}\right)\left(O\left(\langle x\rangle^{-2-t-|\alpha|}\right)\right.$ after differentiation $\left.\partial_{x}^{\alpha} \partial_{\beta}^{\alpha}\right)$, and the last term is $O\left(\langle x\rangle^{-2-2 \varepsilon}\right)\left(O\left(\langle x\rangle^{-2-2 \varepsilon-|x|}\right.\right.$, after $\left.\partial_{x}^{\alpha} \partial_{\xi}^{\beta}\right)$. 
We define $J_{ \pm}=J_{ \pm}(\delta, \Delta)$ by

$$
J_{ \pm}=f\left(\delta / 2,2 \Delta ; H_{0}\right) j_{ \pm}\left(x, D_{x}\right) .
$$

Then the symbol of $J_{ \pm}$concides with $j_{ \pm}$modulo $O\left(\langle x\rangle^{-\infty}\right)$ since $f\left(\delta / 2,2 \Delta ; \xi^{2}\right)=1$ on $\operatorname{supp} j_{ \pm}(\delta, \Delta ; \cdot, \cdot)$.

Lemma 3.2. Let $q_{ \pm}(x, \xi)$ be a symbol such that

$$
\left|\partial_{x}^{\alpha} \partial_{\xi}^{\beta} q_{ \pm}(x, \xi)\right| \leqq\left(\begin{array}{l}
C_{\alpha \beta}\langle x\rangle^{-|\alpha|} \\
C_{\alpha \beta}\langle x\rangle^{-\mu-|\alpha|}( \pm \hat{x} \cdot \hat{\xi} \geqq \gamma)
\end{array}\right.
$$

for any $\alpha, \beta$, with $\gamma \in(-1,1)$ and $\mu \in[0,3]$. Then for $\phi \in \mathscr{D}_{1}$,

$$
\left\|q_{ \pm}\left(x, D_{x}\right) e^{-i t H_{0}} \phi\right\| \leqq C_{\phi}\langle t\rangle^{-\mu}( \pm t \geqq 0)
$$

$C_{\phi}$ depends only on $\phi$ and finite number of constants in the assumption.

Proof. We prove the $(+)$-case only. Let $\tilde{\rho} \in C_{0}^{\infty}((-1,1))$ such that $\tilde{\rho}(\theta)=1$ if $\theta \geqq(1+\gamma) / 2,=0$ if $\theta \leqq \gamma: 0 \leqq \tilde{\rho}(\theta) \leqq 1$, and set

$$
\begin{aligned}
& q_{1}(x, \xi)=q_{+}(x, \xi)\left\{X_{1}(x)+\left(1-X_{1}(x)\right) \cdot \tilde{\rho}(\hat{x} \cdot \hat{\xi})\right\} \\
& q_{2}(x, \xi)=q_{+}(x, \xi)-q_{1}(x, \xi)=q_{+}(x, \xi)\left(1-X_{1}(x)\right)(1-\tilde{\rho}(\hat{x} \cdot \hat{\xi})) .
\end{aligned}
$$

As is easily seen, they satisfy

$$
\begin{gathered}
\left|\partial_{x}^{\alpha} \partial_{\xi}^{\beta} q_{1}(x, \xi)\right| \leqq C_{\alpha \beta}\langle x\rangle^{-\mu-|\alpha|} \\
\left|\partial_{x}^{\alpha} \partial_{\xi}^{\beta} q_{2}(x, \xi)\right| \leqq C_{\alpha \beta}\langle x\rangle^{-|x|} \\
\quad \operatorname{supp} q_{2} \subset\{(x, \xi): \hat{x} \cdot \hat{\xi} \leqq \gamma\} .
\end{gathered}
$$

At first we consider $q_{1}\left(x, D_{x}\right)$ :

$$
q_{1}\left(x, D_{x}\right) e^{-i t H_{0}} \phi=\left(q_{1}\left(x, D_{x}\right)\langle x\rangle^{\mu}\right)\left(\langle x\rangle^{-\mu} e^{-i t H_{0}} \phi\right) \text {. }
$$

and by Lemma 4.3 of [7], we have

$$
\left\|\langle x\rangle^{-\mu} e^{-i t H_{0}} \phi\right\| \leqq C\langle t\rangle^{-\mu},
$$

since $\phi \in \mathscr{D}_{1} \subset D\left(A^{3}\right)$. By virtue of $(3.4), q_{1}\left(x, D_{x}\right)\langle x\rangle^{\mu}$ is bounded in $L^{2}\left(\mathbb{R}^{n}\right)$, and the claim has been proved for $q_{1}$.

Now, (3.5) implies that $q_{2}$ has support in the in-coming subspace, and the Enss method can be applied to obtain

$$
\left\|q_{2}\left(x, D_{x}\right) e^{-i t H_{0}} f\left(\delta, \Delta ; H_{0}\right) \chi_{\{x:|x|<c t\}}\right\| \leqq C_{N}\langle t\rangle^{-N}(t>0)
$$

for any $N$ and sufficiently small $c>0$ (cf. Enss [2]). We take $0<\delta<\Delta<\infty$ so that $f\left(\delta, \Delta ; H_{0}\right) \phi=\phi$. Then

$$
\begin{aligned}
q_{2}\left(x, D_{x}\right) e^{-i t H_{0}} \phi= & \left(q_{2}\left(x, D_{x}\right) e^{-i t H_{0}} f\left(\delta, \Delta ; H_{0}\right) \chi_{\{x:|x|<c t\}}\right) \phi \\
& +\left(q_{2}\left(x, D_{x}\right) e^{-i t H_{0}} f\left(\delta, \Delta ; H_{0}\right)\right)\left(\chi_{\{x:|x| \geqq c t\}} \phi\right)
\end{aligned}
$$


hence we have

$$
\begin{aligned}
\left\|q_{2}\left(x, D_{x}\right) e^{-i t H_{0}} \phi\right\| \leqq & \left\|q_{2}\left(x, D_{x}\right) e^{-i t H_{0}} f\left(\delta, \Delta ; H_{0}\right) \chi_{\{x:|x|<c t\}}\right\|\|\phi\| \\
& +\left\|q_{2}\left(x, D_{x}\right)\right\|\left\|\chi_{\{x|x| \geqq c t\}} \phi\right\| .
\end{aligned}
$$

Since $\phi \in L^{2,3}\left(\mathbb{R}^{n}\right)$,

$$
\left\|\chi_{\{x:|x| \geqq c t\}} \phi\right\| \leqq\langle c t\rangle^{-3}\|\phi\|_{L^{2,3}\left(\mathbb{R}^{n}\right)},
$$

and (3.7), (3.8), (3.9) complete the proof.

Proposition 3.1 For $\phi \in \mathscr{D}_{1}$,

(i) $\left\|\left(H J_{ \pm}-J_{ \pm} H_{0}\right) e^{-i t H_{0}} \phi\right\| \leqq C_{\phi}\langle t\rangle^{-2-\varepsilon}(t \rightarrow \pm \infty)$;

(ii) $\left\|\left(J_{ \pm}^{*} J_{ \pm}-f\left(\delta, \Delta ; H_{0}\right)^{2}\right) e^{-i t H_{0}} \phi\right\| \leqq C_{\phi}\langle t\rangle^{-1-\varepsilon}(|t| \rightarrow \infty)$;

(iii) $\left\|\left(J_{ \pm}-f\left(\delta, \Delta ; H_{0}\right)\right) e^{-i t H_{0}} \phi\right\| \leqq C_{\phi}\langle t\rangle^{-\varepsilon}(|t| \rightarrow \infty)$.

Proof. As remarked after the definition of $J_{ \pm}$, the symbol of $J_{ \pm}$coincides $j_{ \pm}(x, \xi)$ modulo $O\left(\langle x\rangle^{-\infty}\right)$, and we may consider $j_{ \pm}(x, \xi)$ as the symbol of $J_{ \pm}$. Then the symbol of $\left\{\left(H_{0}+V_{1}\right) J_{ \pm}-J_{ \pm} H_{0}\right\}$ is $-t_{ \pm}(x, \xi)$, hence by Lemmas 3.1-(iii) and 3.2, we see

$$
\begin{gathered}
\left.\|\left\{H_{0}+V_{1}\right) J_{ \pm}-J_{ \pm} H_{0}\right\} e^{-i t H_{0}} \phi \| \leqq C\langle t\rangle^{-2-\varepsilon}( \pm t \geqq 0) . \\
V_{2} J_{ \pm} e^{-i t H_{0}} \phi \\
=\left\{V_{2}\langle x\rangle^{2+\varepsilon}\left(H_{0}+1\right)\right\}\left\{\left(H_{0}+1\right)\langle x\rangle^{-2-\varepsilon} J_{ \pm}\langle x\rangle^{2+\varepsilon}\right\}\left\{\langle x\rangle^{-2-\varepsilon} e^{-i t H_{0}} \phi\right\} .
\end{gathered}
$$

The first factor is bounded by Assumption ( $V)$. The symbol of the second factor, say $r(x, \xi)$, satisfies

$$
\left|\partial_{x}^{\alpha} \partial_{\xi}^{\beta} r(x, \xi)\right| \leqq C_{\alpha \beta N}\langle x\rangle^{-|\alpha|}\langle\xi\rangle^{-N}
$$

for any $\alpha, \beta$ and $N$, hence the second factor is bounded. The last factor can be estimated by (3.6) to conclude

$$
\left\|V_{2} J_{ \pm} e^{-i t H_{0}} \phi\right\| \leqq C\langle t\rangle^{-2-\varepsilon} .
$$

This completes the proof of (i).

By the asymptotic expansion theorem $([12], \S 2.3)$ and Lemma 3.1-(ii), the symbol of $\left\{J_{ \pm}^{*} J_{ \pm}-f\left(\delta, \Delta ; H_{0}\right)^{2}\right\}$ is in $S_{1,0}^{-1-\varepsilon}\left(\mathbb{R}_{\xi}^{n}\right)$, so $\left(J_{ \pm}^{*} J_{ \pm}-f\left(\delta, \Delta ; H_{0}\right)^{2}\right)\langle x\rangle^{1+\varepsilon}$ is bounded in $L^{\frac{1}{2}}\left(\mathbb{R}^{n}\right)$. Thus (ii) follows from (3.6) again. (iii) follows similarly from Lemma 3.1-(ii).

Corollary 3.1. For $\phi \in \mathscr{H}$,

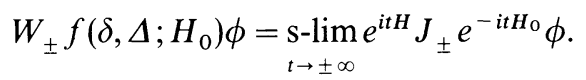

Proof. If $\phi \in \mathscr{D}_{1}$, this follows easily from Proposition 3.1-(iii), and the density argument yields the assertion. 
Corallary 3.2 For $\phi \in \mathscr{D}_{1}$,

(i) $\mid\left(W_{ \pm} f\left(\delta, \Delta ; H_{0}\right)-J_{ \pm}\right) e^{-i t H_{0}} \phi \| \leqq C_{\phi}\langle t\rangle^{-1-\varepsilon}( \pm t \geqq 0)$;

(ii) $\int_{0}^{ \pm \infty}\left\|\left(W_{ \pm} f\left(\delta, \Delta ; H_{0}\right)-J_{ \pm}\right) e^{-i t H_{0}} \phi\right\| d t<\infty$.

Proof. We prove the $(+)$-case only. By Corollary 3.1,

$$
\begin{aligned}
\left(W_{ \pm}\right. & \left.f\left(\delta, \Delta ; H_{0}\right)-J_{+}\right) e^{-i t H_{0}} \phi=\left(\underset{s-\lim }{s \rightarrow+\infty} e^{i s H} J_{+} e^{-i s H_{0}}-J_{+}\right) e^{-i t H_{0}} \phi \\
& =\int_{0}^{\infty} i e^{i s H}\left(H J_{+}-J_{+} H_{0}\right) e^{-i t H_{0}} e^{-i t H_{0}} \phi d s .
\end{aligned}
$$

Hence we have

$$
\begin{aligned}
\left\|\left(W_{+} f\left(\delta, \Delta ; H_{0}\right) J_{+}\right) e^{-i t H_{0}} \phi\right\| & \leqq \int_{0}^{\infty}\left\|\left(H J_{+}-J_{+} H_{0}\right) e^{-i(s+t) H_{0}} \phi\right\| d s \\
& \leqq \int_{0}^{\infty} C|s+t|^{-2-\varepsilon} d s=\frac{C}{2+\varepsilon}|t|^{-1-\varepsilon} \quad(t>0)
\end{aligned}
$$

by Proposition 3.1-(i). (ii) follows immediately from (i).

\section{Proof Theorem 1.}

At first, we sum up the remainder terms of (2.1).

Lemma 4.1. As forms on $H^{2}\left(\mathbb{R}^{n}\right)$,

$$
\begin{gathered}
X_{R} H_{0}=\frac{i}{2}\left[H_{0}, A_{R}\right]+X_{R} H_{0}\left(1-Z_{R}\right)-C_{R} ; \\
X_{R} H=\frac{i}{2}\left[H, A_{R}\right]+X_{R} V-\frac{i}{2}\left[V, A_{R}\right]+X_{R} H_{0}\left(1-Z_{R}\right)-C_{R} .
\end{gathered}
$$

Equation (4.1) follows from the definitions of $A_{R}$ and $C_{R}$. Equation (4.2) follows immediately from (4.1).

We fix $\phi, \psi \in \mathscr{D}_{1} \cap\left(S^{-1} \mathscr{D}_{1}\right)$ and $0<\delta<\Delta<\infty$ so that $f\left(\delta, \Delta ; H_{0}\right) \phi=\phi$, $f\left(\delta, \Delta ; H_{0}\right) \psi=\psi$. Then we obtain by Lemma 4.1,

$$
\begin{aligned}
\left(e^{-i t H} W_{-} \phi, X_{R} e^{-i t H} W_{-} H_{0} \psi\right)-\left(e^{-i t H_{0}} \phi, X_{R} e^{-i t H_{0}} H_{0} \psi\right) \\
=\left(\phi(t), X_{R} H \psi(t)\right)-\left(\phi_{0}(t), X_{R} H_{0} \psi_{0}(t)\right) \\
\left.=\frac{i}{2}\left\{\left(\phi(t),\left[H, A_{R}\right] \psi(t)\right)-\left(\phi_{0}(t),\left[H_{0}, A_{R}\right] \psi_{0} t\right)\right)\right\} \\
\quad+\left(\phi(t),\left\{X_{R} V+\frac{i}{2}\left[A_{R}, V\right]\right\} \psi(t)\right)+\left(\phi(t), X_{R} H_{0}\left(1-Z_{R}\right) \psi(t)\right) \\
\quad-\left(\phi_{0}(t), X_{R} H_{0}\left(1-Z_{R}\right) \psi_{0}(t)\right)-\left\{\left(\phi(t), C_{R} \psi(t)\right)-\left(\phi_{0}(t), C_{R} \psi_{0}(t)\right)\right\} .
\end{aligned}
$$

We shall estimate the integrals of these terms. 
Lemma 4.2. For sufficiently large $R$,

$$
\lim _{\substack{T \rightarrow \infty \\ T^{\prime} \rightarrow-\infty}} \int_{T^{\prime}}^{T}\left\{\left(\phi(t),\left[H, A_{R}\right] \psi(t)\right)-\left(\phi_{0}(t),\left[H_{0}, A_{R}\right] \psi_{0}(t)\right)\right\} d t=0 .
$$

Proof. By (1.3), we have

$$
\begin{gathered}
i\left\{\left(\phi(t),\left[H, A_{R}\right] \psi(t)\right)-\left(\phi_{0}(t),\left[H_{0}, A_{R}\right] \psi_{0}(t)\right)\right\} \\
=\frac{d}{d t}\left\{\left(\phi(t), A_{R} \psi(t)\right)-\left(\phi_{0}(t), A_{R} \psi_{0}(t)\right)\right\},
\end{gathered}
$$

hence

$$
\begin{aligned}
i \int_{T^{\prime}}^{T}\left\{\left(\phi(t),\left[H, A_{R}\right] \psi(t)\right)-\left(\phi_{0}(t),\left[H_{0}, A_{R}\right] \psi_{0}(t)\right)\right\} d t \\
=\left\{\left(\phi(T), A_{R} \psi(T)\right)-\left(\phi_{0}(T), A_{R} \psi_{0}(T)\right)\right\} \\
\quad-\left\{\left(\phi\left(T^{\prime}\right), A_{R} \psi\left(T^{\prime}\right)\right)-\left(\phi_{0}\left(T^{\prime}\right), A_{R} \psi_{0}\left(T^{\prime}\right)\right)\right\} .
\end{aligned}
$$

Again in by (1.3),

$$
\begin{aligned}
& \left(\phi(t), A_{R} \psi(t)\right)-\left(\phi_{0}(t), A_{R} \psi_{0}(t)\right) \\
& \quad=\left(W_{-} e^{-i t H_{0}} \phi, A_{R} W_{-} e^{-i t H_{0}} \psi\right)-\left(e^{-i t H_{0}} \phi, A_{R} e^{-i t H_{0}} \psi\right) \\
& \quad=\left(\left(W_{-}-1\right) e^{-i t H_{0}} \phi, A_{R} W_{-} e^{-i t H_{0}} \psi\right)+\left(A_{R}^{*} e^{-i t H_{0}} \phi,\left(W_{-}-1\right) e^{-i t H_{0}} \psi\right) .
\end{aligned}
$$

Since $A_{R}, A_{R}^{*} \in B\left(H^{1}\left(\mathbb{R}^{n}\right), L^{2}\left(\mathbb{R}^{n}\right)\right)$ for each $R \quad$ (see Lemma 2.2) and $\left\|\left(W_{-}-1\right) \exp \left(-i t H_{0}\right) \phi\right\| \rightarrow 0(t \rightarrow-\infty)$ by definition of $W_{-}$, we obtain

$$
\begin{aligned}
& \left|\left(\phi(t), A_{R} \psi(t)\right)-\left(\phi_{0}(t), A_{R} \psi_{0}(t)\right)\right| \\
& \leqq\left\|\left(W_{-}-1\right) e^{-i t H_{0}} \phi\right\|\left\|A_{R}\right\|_{B\left(H^{1}, L^{2}\right)}\left\|W_{-}\right\|_{B\left(H^{2}\right)}\|\psi\|_{H^{2}} \\
& +\left\|A_{R}^{*}\right\|_{B\left(H^{1}, L^{2}\right)}\|\phi\|_{H^{1}}\left\|\left(W_{-}-1\right) e^{-i t H_{0}} \psi\right\| \\
& \longrightarrow 0 \quad(t \rightarrow-\infty) \text {. }
\end{aligned}
$$

We will show

$$
\left(\phi(t), A_{R} \psi(t)\right)-\left(\phi_{0}(t), A_{R} \psi_{0}(t)\right) \rightarrow 0 \quad(t \rightarrow \infty) .
$$

Let $\phi_{1}(t)$ and $\psi_{1}(t)$ be

$$
\phi_{1}(t)=\exp \left(-i t H_{0}\right) S \phi ; \quad \psi_{1}(t)=\exp \left(-i t H_{0}\right) S \psi .
$$

Then similarly to (4.5) and (4.6), one can see

$$
\left(\phi(t), A_{R} \psi(t)\right)-\left(\phi_{1}(t), A_{R} \psi_{1}(t)\right) \rightarrow 0 \quad(t \rightarrow \infty) .
$$

If $R$ is so large that $2 / R \leqq \delta$, then $\xi \in \operatorname{supp} \hat{\psi},|x| \geqq 2 R$ and $\hat{x} \cdot \hat{\xi} \geqq 1 / 4$ imply $a_{R}(x, \xi) Z_{R}(\xi)=R^{\prime}|\xi|$ by Lemma 2.1 , where $R^{\prime}=$ constant $\times R$ in Lemma 2.1 . Therefore, using Lemma 3.2, one can show

$$
\left\|\left(A_{R}-R^{\prime}\left|D_{x}\right|\right) e^{-i t H_{0}} \psi\right\| \rightarrow 0(t \rightarrow \infty) .
$$


It follows that

$$
\begin{aligned}
\left(\phi_{0}(t), A_{R} \psi_{0}(t)\right)= & \left(\phi_{0}(t), R^{\prime}\left|D_{x}\right| \psi_{0}(t)\right)+\left(\phi_{0}(t),\left(A_{R}-R^{\prime}\left|D_{x}\right|\right) e^{-i t H_{0}} \psi\right) \\
= & R^{\prime}\left(\phi,\left|D_{x}\right| \psi\right)+\left(\phi_{0}(t),\left(A_{R}-R^{\prime}\left|D_{x}\right|\right) e^{-i t H_{0}} \psi\right) \\
& \longrightarrow R^{\prime}\left(\phi,\left|D_{x}\right| \psi\right) \quad(t \rightarrow \infty) .
\end{aligned}
$$

In the same way, we have

$$
\begin{aligned}
& \left(\phi_{1}(t), A_{R} \psi_{1}(t)\right) \\
& \quad \longrightarrow R^{\prime}\left(S \phi,\left|D_{x}\right| S \psi\right)=R^{\prime}\left(\phi,\left|D_{x}\right| \psi\right) \quad(t \rightarrow \infty) .
\end{aligned}
$$

Combining (4.8) with (4.9) and (4.10), we obtain (4.7). The lemma follows from (4.4), (4.6) and (4.7).

Lemma 4.3. For sufficiently large $R$,

$$
\int_{-\infty}^{\infty}\left(\phi_{0}(t), X_{R} H_{0}\left(1-Z_{R}\right) \psi_{0}(t)\right) d t=0 .
$$

Proof. This is immediate since $\left(1-Z_{R}\right) \psi=0$ if $2 / R \leqq \delta$.

\section{Lemma 4.4}

$$
\lim _{R \rightarrow \infty} \int_{-\infty}^{\infty}\left(\phi(t), X_{R} H_{0}\left(1-Z_{R}\right) \psi(t)\right) d t=0
$$

where the integral converges absolutely.

Proof. Since the integrand clearly converges to zero for each $t$, it is sufficient to show that the integral is dominated uniformly.

Let $M_{R}=X_{R} H_{0}\left(1-Z_{R}\right)$. Then, similarly to (4.4) and (4.6), we see

$$
\begin{aligned}
& \left|\left(\phi(t), M_{R} \psi(t)\right)\right| \\
& \leqq\left[\begin{array}{l}
\left|\left(\phi_{0}(t), J_{-}^{*} M_{R} J_{-} \psi_{0}(t)\right)\right|+\left\|\left(W_{-}-J_{-}\right) e^{-i t H_{0}} \phi\right\|\left\|M_{R}\right\|\|\psi\| \\
+\left\|J_{-}^{*} M_{R}^{*}\right\|\|\phi\|\left\|\left(W_{-}-J_{-}\right) e^{-i t H_{0}} \psi\right\| \\
\left|\left(\phi_{1}(t), J_{+}^{*} M_{R} J_{+} \psi_{1}(t)\right)\right|+\left\|\left(W_{+}-J_{+}\right) e^{-i t H_{0}} S \phi\right\|\left\|M_{R}\right\|\|\psi\| \\
+\left\|M_{R}^{*} J_{+}\right\|\|\phi\|\left\|\left(W_{+}-J_{+}\right) e^{-i t H_{0}} S \psi\right\| .
\end{array}\right.
\end{aligned}
$$

If $2 / R \leqq \delta / 4, M_{R} J_{ \pm}=0$ and by Corollary 3.2 , we have

$$
\left|\left(\phi(t), M_{R} \psi(t)\right)\right| \leqq C\langle t\rangle^{-1-\varepsilon}
$$

to conclude the assertion.

\section{Lemma 4.5}

$$
\lim _{R \rightarrow \infty} \int_{-\infty}^{\infty}\left\{\left(\phi(t), C_{R} \psi(t)\right)-\left(\phi_{0}(t), C_{R} \psi_{0}(t)\right)\right\} d t=0
$$

where the integral converges absolutely.

Proof. By Lemma 2.3, $C_{R}$ weakly converges to zero and it is sufficient to show the dominated convergence, again. 
On $(-\infty, 0)$, the integrand is

$$
\begin{aligned}
& \left(\phi(t), C_{R} \psi(t)\right)-\left(\phi_{0}(t), C_{R} \psi_{0}(t)\right)=\left(\left(W_{-}-J_{-}\right) e^{-i t H_{0}} \phi, C_{R} W_{-} e^{-i T H_{0}} \psi\right) \\
& \quad+\left(C_{R}^{*} J_{-} e^{-i t h_{0}} \phi,\left(W_{-}-J_{-}\right) e^{-i t H_{0}} \psi\right)+\left(e^{-i t H_{0}} \phi,\left(J_{-}^{*} C_{R_{-}} J_{-}-C_{R}\right) e^{-i t H_{0}} \psi\right),
\end{aligned}
$$

and the former two terms can be dominated in the same way as the last lemma using Proposition 2.2. We have

$$
J_{-}^{*} C_{R} J_{-}-C_{R}=J_{-}^{*}\left[C_{R}, J_{-}\right]+\left(J_{-}^{*} J_{-}-1\right) C_{R}
$$

and by Lemmas 2.2, 3.1 and the asymptotic expansion theorem, the symbol of $\left[C_{R}, J_{-}\right]$satisfy the assumption of Lemma 3.2 with $\mu=-1-\varepsilon((-)$-case) uniformly in $R$, if $|\xi| \geqq \delta$. Hence, by Lemma 3.2, Proposition 3.1-(ii) and Proposition 2.2-(iii),

$$
\begin{aligned}
& \left|\left(e^{-i t H_{0}} \phi,\left(J_{-}^{*} C_{R} J_{-}-C_{R}\right) e^{-i t H_{0}} \psi\right)\right| \leqq\left\|J_{-} e^{-i t H_{0}} \phi\right\|\left\|\left[C_{R}, j_{-}\right] e^{-i t H_{0}} \psi\right\| \\
& \quad+\left\|\left(J_{-}^{*} J_{-}-1\right) e^{-i t H_{0}} \phi\right\|\left\|C_{R} e^{-i t H_{0}} \psi\right\| \leqq C\langle t\rangle^{-1-\varepsilon} \quad(t \leqq 0) .
\end{aligned}
$$

It follows immediately from Lemma 2.2 that the symbol of $C_{R} f\left(\delta, \Delta ; H_{0}\right)$ satisfies the assumption of Lemma 3.2 with $\mu=2((+)$-case) uniformly in $R$ and

$$
\left\|C_{R} e^{-i t H_{0}} \psi\right\| \leqq C\langle t\rangle^{-2}(t \geqq 0) .
$$

Since

$$
\begin{aligned}
\left(\phi(t), C_{R} \psi(t)\right)= & \left(\left(W_{+}-J_{+}\right) e^{-i t H_{0}} S \phi, C_{R} \dot{W}_{+} \psi_{1}(t)\right) \\
& +\left(C_{R}^{*} J_{+} \phi_{1}(t),\left(W_{+}-J_{+}\right) e^{-i t H_{0}} S \psi\right) \\
& +\left(J_{+} \phi_{1}(t),\left(C_{R} J_{+}\right) e^{-i t H_{0}} S \psi\right),
\end{aligned}
$$

and the symbol of $\left(C_{R} J_{+}\right)$satisfies the same estimates as Lemma 2.2-(ii), we can conclude by Lemma 3.2 and Corollary 3.2,

$$
\begin{aligned}
& \left|\left(\phi(t), C_{R} \psi(t)\right)\right| \leqq\left\|\left(W_{+}-J_{+}\right) e^{-i t H_{0}} S \phi\right\|\left\|C_{R} W_{+} \psi_{1}(t)\right\| \\
& \quad+\left\|C_{R}^{*} J_{+} \phi_{1}(t)\right\|\left\|\left(W_{+}-J_{+}\right) e^{-i t H_{0}} S \psi\right\|+\left\|J_{+} \phi(t)\right\|\left\|\left(C_{R} J_{+}\right) e^{-i t H_{0}} S \psi\right\| \\
& \quad \leqq C\langle t\rangle^{-1-\varepsilon} \quad(t \geqq 0) .
\end{aligned}
$$

Thus the integrand is dominated uniformly in $R$.

\section{Lemma 4.6.}

$$
\lim _{R \rightarrow \infty} \int_{-\infty}^{\infty}\left(\phi(t),\left\{X_{R} V+\frac{i}{2}\left[A_{R}, V\right]\right\} \psi(t)\right) d t=\int_{-\infty}^{\infty}\left(\phi(t),\left\{V+\frac{i}{2}[A, V]\right\} \psi(t)\right) d t,
$$

where the integrals converge absolutely.

Proof. For each $t$, clearly

$$
\lim _{R \rightarrow \infty}\left(\phi(t), X_{R} V \psi(t)\right)=(\phi(t), V \psi(t)),
$$

and by Proposition 2.2 and Lemma 2.3,

$$
\begin{aligned}
\left(\phi(t),\left[A_{R}, V\right] \psi(t)\right)= & \left(A_{R}^{*} \phi(t), V \psi(t)\right)-\left(V \phi(t), A_{R} \psi(t)\right) \\
= & \left(\left(\langle x\rangle^{-1} A_{R}^{*}\right) \phi(t),(\langle x\rangle V) \psi(t)\right) \\
& -\left((\langle x\rangle V) \phi(t),\left(\langle x\rangle^{-1} A_{R}\right) \psi(t)\right)
\end{aligned}
$$




$$
\begin{gathered}
\underset{R \rightarrow \infty}{\longrightarrow}\left(\langle x\rangle^{-1}\left(-\frac{1}{i} x \cdot \nabla-\frac{i}{2}(n-2)\right) \phi(t),(\langle x\rangle V) \psi(t)\right) \\
-\left(\langle x\rangle V \phi(t),\langle x\rangle^{-1}\left(-\frac{1}{i} x \cdot \nabla+\frac{i}{2}(n-2)\right) \psi(t)\right) \\
=(\phi(t),[A, V] \psi(t)) .
\end{gathered}
$$

It remains to prove the dominated convergence.

Since $V$ is locally $H$-smooth, we have

$$
\int_{-\infty}^{\infty}\left|\left(\phi(t), X_{R} V \psi(t)\right)\right| d t \leqq C_{\phi \psi}<\infty .
$$

Similarly to (4.11), we obtain

$$
\begin{aligned}
& \langle x\rangle V_{2} \phi(x)=\left\{\langle x\rangle V_{2}(H+i)^{-1}\right\} e^{-i t H} W_{-}\left(H_{0}+i\right) \phi \\
& =\left[\begin{array}{l}
\left\{\langle x\rangle V_{2}(H+i)^{-1}\right\}\left\{J_{-} e^{-i t H_{0}}\left(H_{0}+i\right) \phi+\left(W_{-}-J_{-}\right) e^{-i t H_{0}}\left(H_{0}+i\right) \phi\right\} \\
\left\{\langle x\rangle V_{2}(H+i)^{-1}\right\}\left\{J_{+} e^{-i t H_{0}} S\left(H_{0}+i\right) \phi+\left(W_{+}-J_{+}\right) e^{-i t H_{0}} S\left(H_{0}+i\right) \phi\right\} .
\end{array}\right. \\
& \|\langle x\rangle V \phi(t)\| \\
& \leqq\left[\begin{array}{c}
\left\|\langle x\rangle V_{2}(H+i)^{-1}\langle x\rangle^{1+\varepsilon}\right\|\left\|\langle x\rangle^{-1-\varepsilon} J e^{-i t H_{0}}\left(H_{0}+i\right) \phi\right\| \\
+\left\|\langle x\rangle V_{2}(H+i)^{-1}\right\|\left\|\left(W_{-}-J_{-}\right) e^{-i t H_{0}}\left(H_{0}+1\right) \phi\right\| \\
\left\|\langle x\rangle V_{2}(H+i)^{-1}\langle x\rangle^{1+\varepsilon}\right\|\left\|\langle x\rangle^{-1-\varepsilon} J_{+} e^{-i t H_{0}} S\left(H_{0}+i\right) \phi\right\| \\
+\left\|\langle x\rangle V_{2}(H+i)^{-1}\right\|\left\|\left(W_{+}-J_{+}\right) e^{-i t H_{0}} S\left(H_{0}+i\right) \phi\right\| .
\end{array}\right.
\end{aligned}
$$

Lemma 3.2 can be applied to $\left(\langle x\rangle^{-1-\varepsilon} J_{ \pm}\right)$, and combining this with Corollary 3.2 we conclude

$$
\left\|\langle x\rangle V_{2} \phi(t)\right\| \leqq C\langle t\rangle^{-1-\varepsilon} \quad(t \in \mathbb{R})
$$

This implies

$$
\left|\left(\phi(t),\left[A, V_{2}\right] \psi(t)\right)\right| \leqq C\langle t\rangle^{-1-\varepsilon} \quad(t \in \mathbb{R})
$$

by virtue of (4.12);

$$
\begin{aligned}
\left(\phi(t),\left[A_{R}, V_{1}\right] \psi(t)\right)= & \left(\left(W_{ \pm}-J_{ \pm}\right) \phi_{i}(t),\left[A_{R}, V_{1}\right] \psi(t)\right) \\
& +\left(J_{ \pm} \phi_{i}(t),\left[A_{R}, V_{1}\right]\left(W_{ \pm}-J_{ \pm}\right) \psi_{i}(t)\right) \\
& +\left(J_{ \pm} \phi_{i}(t),\left[A_{R}, V_{1}\right] J_{ \pm} \psi_{i}(t)\right) .
\end{aligned}
$$

where $i=1 / 0$ for $(+) /(-)$ respectively. The former two terms can be dominated as above (we remark that $\left[A_{R}, V_{1}\right]=A_{R} V_{1}-V_{1} A_{R}$ is uniformly bounded in $B\left(H^{1}, L^{2}\right)$ ).

$$
\left[A_{R}, V_{1}\right] J_{ \pm}=\left[A_{R} J_{ \pm}, V_{1}\right]+A_{R}\left[V_{1}, J_{ \pm}\right] \text {. }
$$

The symbol of $\left[A_{R} J_{ \pm}, V_{1}\right]\left(\left[V_{1}, J_{ \pm}\right]\right.$respectively) is in $S_{1,0}^{-1-\varepsilon}\left(\mathbb{R}_{\xi}^{n}\right)\left(S_{1,0}^{-2-2 \varepsilon}\left(\mathbb{R}_{\xi}^{n}\right)\right.$ respectively), and is bounded in $R$ by Lemmas 2.2, 3.1 and Assumption ( $V)$. Hence

$$
\begin{aligned}
\left|\left(J_{ \pm} \phi_{i}(t),\left[A_{R}, V_{1}\right] J_{ \pm} \psi_{i}(t)\right)\right| \leqq & \left\|J_{ \pm} \phi_{i}(t)\right\|\left\|\left[A_{R} J_{ \pm}, V_{1}\right] \psi_{i}(t)\right\| \\
& +\left\|A_{R}^{*}\right\|_{B\left(H^{1}, L^{2,-1}\right)}\left\|J_{ \pm} \phi_{i}(t)\right\|_{H^{1}}\left\|\langle x\rangle\left[V_{1}, J_{ \pm}\right] \psi_{i}(t)\right\| \\
\leqq & C\langle t\rangle^{-1-\varepsilon}(t \in \mathbb{R}),
\end{aligned}
$$


and these estimates follow

$$
\left|\left(\phi(t),\left[A_{R}, V_{1}\right] \psi(t)\right)\right| \leqq C\langle t\rangle^{-1-\varepsilon}(t \in \mathbb{R}) .
$$

Equations (4.13) and (4.14) prove the dominated convergence.

Proof of Theorem 1. Combining (4.3) with Lemmas 4.2-4.6, we obtain

$$
\begin{aligned}
\lim _{R \rightarrow \infty}\left(\phi, T_{R} H_{0} \psi\right) & =\lim _{R \rightarrow \infty}\left\{\int_{-\infty}^{\infty}\left(\phi(t), X_{R} H \psi(t)\right) d t-\int_{-\infty}^{\infty}\left(\phi_{0}(t), X_{R} H_{0} \psi_{0}(t)\right) d t\right\} \\
& =\int_{-\infty}^{\infty}\left(\phi(t),\left\{V+\frac{i}{2}[A, V]\right\} \psi(t)\right) d t .
\end{aligned}
$$

Acknowledgements. The author wishes to thank Professor K. Yajima and Professor H. Kitada for valuable discussions. In particular, Professor Kitada informed about the time-delay operator and Professor Yajima suggested to employ pseudo-differential operators. The author also thanks Professor S. T. Kuroda for various support.

\section{References}

1. Amrein, W. O., Jauch, J. M., Sinha, K. B.: Scattering theory in quantum mechanics. Reading, MA: W. A. Benjamin 1977

2. Enss, V.: Geometric methods in spectral and scattering theory. In: Velo G., Wightman, A. S. (eds.). Rigorous atomic and molecular physics. pp. 1-69. New York: Plenum Press 1981

3. Hörmander, L.: The analysis of linear partial differential operators. Vol. I-IV. Berlin, Heidelberg, New York: Springer 1983-1985

4. Isozaki, H., Kitada, H.: Scattering matrices for two-body Schrödinger operators. Sci. Pap. Col. Arts and Sci., Univ. Tokyo 35, 81-107 (1985)

5. Jauch, J. M., Marchand, J. P.: The delay time operator for simple scattering systems. Helv. Phys. Acta 40, 217-229 (1967)

6. Jauch, J. M., Sinha, K. B., Misra, B. N.: Time-delay in scattering processes. Helv. Phys. Acta 45, 398426 (1972)

7. Jensen, A.: Time-delay in potential scattering theory. Some "geometric" results. Commun. Math. Phys. 82, 435-456 (1981)

8. Jensen, A.: On Lavine's formula for time-delay. Math. Scand. 54, 253-261 (1984)

9. Jensen, A.: A stationary proof of Lavine's formula for time-delay. Lett. Math. Phys. 7, 137-143 (1983)

10. Lavine, R.: Commutators and local decay. In: Lavita, J. A., Marchand J. P. (eds.). Scattering theory in mathematical physics. pp. 141-156. Dordrecht: Reidel 1974

11. Read, M., Simon, B.: Methods of modern mathematical physics, Vol. I-IV. New York: Academic Press 1971-1978

12. Taylor, M.: Pseudodifferential operators. Princeton Math. Series, New Jersey: Princeton University Press 1981

13. Martin, Ph.: Time-delay of quantum scattering process. Acta Phys. Austr. Suppl. 23, 157-208 (1981)

14. Wang, X P.: Opérateurs de temps-retards dans la théorie de la diffuson. CR. Acad. Sci. Paris. Sér. I, 301, 789-791 (1985)

15. Wang, X. P.: Low energy resolvent estimates and continuity of time-delay operators. preprint, University de Rennes I

16. Wang, X. P.: Phase-space description of time-delay in scattering theory. Preprint, University de Nantes

Communicated by B. Simon

Received July 25, 1986, in revised form September 23, 1986 
